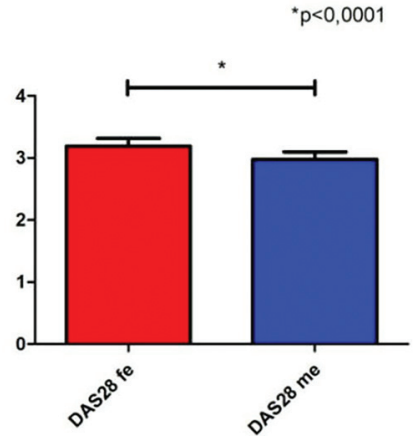

fe: female examiner me: male examiner

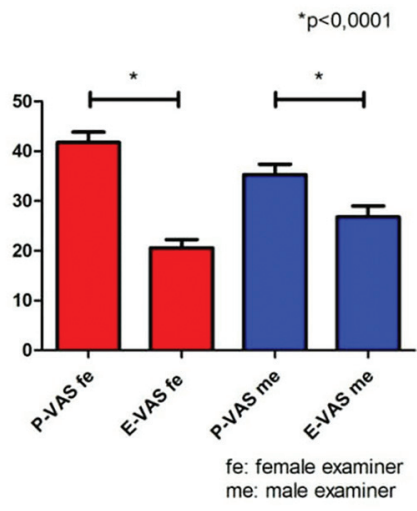

Figure 1

Disclosure of Interests: None declared DOI: 10.1136/annrheumdis-2019-eular.6980

\section{FRI0041 INCIDENCE AND TREND OF PROGRESSIVE MULTIFOCAL LEUKOENCEPHALOPATHY (PML) IN PATIENTS WITH RHEUMATOID ARTHRITIS IN SPAIN. (TREND-AR STUDY)}

María Espinosa ${ }^{1}$, Alberto García-Vadillo², Carmen Barbadillo ${ }^{3}$, Natalia CrespíVillarías $^{4}$, Manuel Fernandez-Prada ${ }^{5}$, María Galindo-Izquierdo ${ }^{6}$, Hilda Godoy ${ }^{3}$, Angeles Herranz Varela ${ }^{7}$, Cristina Macía-Villa ${ }^{8}$, Concepción Morado Quiroga ${ }^{9}$, Jose Luis Morell Hita ${ }^{10}$, Marina Peña ${ }^{11}$, Elia Perez-Fernandez ${ }^{12}$, Javier Quirós ${ }^{13}$ Virginia Villaverde ${ }^{14}$, Cristina Martinez-Prada ${ }^{9}$, Ramón Mazzucchelli ${ }^{13}$. ${ }^{1}$ Infanta Sofia University Hospital, Rheumatology, San Sebastián de los Reyes, Spain; ${ }^{2}$ Hospital de La Princesa, Rheumatology, Madrid, Spain; ${ }^{3}$ Hospital Puerta de Hierro-Majadahonda, Rheumatology, Majadahonda, Spain; ${ }^{4}$ Health Center Rivota, Alcorcón, Spain; ${ }^{5}$ Hospital Universitario de Guadalajara, Guadalajara, Spain; ${ }^{6}$ Hospital 12 De Octubre, Madrid, Spain; ${ }^{7}$ Hospital Henares, Rheumatology, Coslada, Spain; ${ }^{8}$ Hospital Universitario Severo Ochoa, Rheumatology, Leganés, Spain; ${ }^{9}$ Hospital Clinico Universitario San Carlos, Rheumatology, Madrid, Spain; ${ }^{10}$ Hospital Ramón YCajal, Madrid, Spain; ${ }^{11}$ Fundación Hospital Alcorcón, Rehabilitation, Alcorcón, Spain; ${ }^{12}$ Fundación Hospital Alcorcón, Clinical Investigation Unit, Alcorcón, Spain; ${ }^{13}$ Hospital Universitario Fundación Alcorcón, Rheumatology, Alcorcón, Spain; ${ }^{14}$ Hospital de Móstoles, Rheumatology, Móstoles, Spain

Background: Treatment with biological agents is recognized as a potential risk factor for the development of Progressive Multifocal Leukoencephalopathy (PML), a rare and often fatal demyelinating disease of the CNS caused by John Cunningham Virus infection.

Objectives: Analyze the incidence and trend of hospital admissions for PML in patients with RA, in Spain, during the period between 1999 and 2015.

Methods: Population study based on the analysis of a national administrative database that includes a Minimum Basic Data Set (MBDS) of the income of patients with RA (ICD 9 714). Period: January 1, 1999, to December 31,2015 . The cases of PML were identified due to the presence in the primary and secondary diagnosis of the codes ICD 9046.0 to 046.9. The population at risk was estimated through the population census of the National Institute of Statistics, with an estimated prevalence of RA of $0.5 \%$ in both sexes $(0.2 \%$ in men and $0.8 \%$ in women). Crude and adjusted rates were calculated at national level. The trend was analyzed using Generalized Linear Models (MLG) using the variable year as the analysis variable.

Results: Of the total of 338,343 hospital admissions in patients with RA during the 17 years of the study period, only 14 cases $(0.004 \%)$ of $\mathrm{PML}$ were recorded, nine (64.3\%) were women and $5(35.7 \%)$ men. The mean age was 69.5 years (SD 16.8); 74.56 (SD14.8) in women and 60 (SD18.1) in men $(p=0.145)$. Five patients $(35.7 \%)$ died during admission ( 2 women and 3 men) $(p=0.266)$. The average of the Charlson index was 2.21 (SD 1.7); 3 (SD 2.5) in women and 1.78 (SD 1.1) in men $(p=0.227)$.

The crude incidence rate of PML was $0.54 / 100,000$ inhabitants * year, $0.85 / 100,000$ inhabitants * year in men and $0.37 / 100,000$ inhabitants * year in women. The Relative Risk male: female was 2.34. The gross rate of $\mathrm{PML}$ increased from $0.32 / 105$ * year in the period 1999-2002, to 0.74 between 2011 and 2015, both in women (from 0.19 in the period 1999-2002 to 0.52 from 2011 to 2015) as well as men (from 0.82 in the period 1999-2002 to 1.64 during 2011-2015). It is estimated that this increase is of $19.7 \%$ per year.

Conclusion: In Spain, between 1999 and 2015, there has been an increase in the incidence rate of PML in patients with RA. We estimate an annual increase of $19,7 \%$.

Disclosure of Interests: None declared

DOI: 10.1136/annrheumdis-2019-eular.2478

\section{FRI0042 CERTOLIZUMAB PEGOL EFFECTIVENESS IN WOMEN OF CHILDBEARING AGE WITH RHEUMATOID ARTHRITIS: RETROSPECTIVE ANALYSIS OF AN INTERNATIONAL MULTICENTRE COHORT}

Ennio Giulio Favalli ${ }^{1}$, Andrea Becciolini ${ }^{1}$, Roberto Caporali ${ }^{2}$, Piercarlo Sarzi Puttini ${ }^{3}$, Roberto Gorla ${ }^{4}, R$ Ionescu ${ }^{5}$, S Rednic $^{6}$, A Balanescu $^{5}$, E Rezus ${ }^{7}$ C Mogosan ${ }^{5}$, Catalin Codreanu ${ }^{5} .{ }^{1}$ LORHEN Registry and Gaetano Pini-CTO Institute, Rheumatology, Milano, Italy, ${ }^{2}$ LORHEN Registry and University of Pavia, IRCCS Policlinico San Matteo, Rheumatology, Pavia, Italy; ${ }^{3}$ LORHEN Registry and L. Sacco Hospital, Rheumatology, Milano, Italy, ${ }^{4}$ LORHEN Registry and ASST Spedali Civili, Rheumatology, Brescia, Italy, ${ }^{5}$ RRBR Registry and University of Medicine and Pharmacy "Carol Davila" Bucharest, Bucarest, Romania; ${ }^{6}$ RRBR Registry and University of Medicine and Pharmacy "Iuliu Hatieganu" Cluj, Rheumatology, Cluj, Romania; ${ }^{7} R R B R$ Registry and University of Medicine and Pharmacy "Grigore T. Popa" lasi, lasi, Romania

Background: Even though certolizumab pegol (CZP) has been licensed for the treatment of rheumatoid arthritis (RA) a long time ago, observational data in real-life settings are still lacking. Moreover, recent data on the lack of transplacental passage (1) make CZP particularly appealing for the treatment of RA women with a desire of pregnancy.

Objectives: To evaluate in a real-life international cohort the frequency of use, the clinical response, and the retention rate of CZP in RA women of childbearing age.

Methods: Data were retrospectively extracted from the Italian LORHEN and the Romanian Registry of Rheumatic Diseases (RRBR) registries, which include all RA patients treated with CZP between December 2010 and October 2018. The analysis was limited to women who received CZP as first-line biologic agent. The study population was stratified according to childbearing age (18-49 versus $>49$ years). The $6-, 12-$ and 24-month clinical response was evaluated as the proportion of patients achieving Disease Activity Score 28 (DAS28) remission and compared between the subgroups by a chi-squared test. The 5-year retention rate was calculated by the Kaplan-Meier method and compared between the subgroups by log-rank test.

Results: The whole cohort included 630 RA patients treated with CZP According to the inclusion criteria, the study population consisted of 308 female RA patients (mean $[ \pm$ standard deviation, SD] age $54.2 \pm 12.1$ years; mean disease duration $8.7 \pm 8.4$ years; baseline DAS28 $5.25 \pm 1.72$, positive anti-citrullinated peptide antibody 161/254 [63.4\%], positive rheumatoid factor $213 / 297$ [71.7\%]). Apart from mean age (39.6 6.6 vs 60.8 \pm 7.2 , respectively; $p<0.001$ ), no other significant differences in baseline characteristics between the childbearing $(n=97,31.5 \%)$ and non-childbearing age $(n=211,68.5 \%)$ groups were observed. In the overall population, DAS28 remission was achieved by $29.9 \%, 42.1 \%$, and $39.7 \%$ of patients at 6,12 , and 24 months, respectively. This proportion was significantly higher in childbearing compared with non-childbearing women at each timepoint $(39.4 \%$ vs $25.4, p=0.02$ at 6 months; $52.8 \%$ vs $37 \%, p=0.014$ at 12 months; $52 \%$ vs $34.2 \%, p=0.014$ at 24 months). The overall 5 -year retention rate was $37.1 \%$, with a higher (but not statistically significant) persistence in the childbearing versus non-childbearing subgroup $(55.1 \%$ 